\title{
ACIANTHERA HYGROPHILA (ORCHIDACEAE), NUEVO REGISTRO PARA URUGUAY
}

\author{
ANDRÉS J. ROSSADO ${ }^{1}$, PATRICIA L. MAI ${ }^{2}$, JOSÉ M. BONIFACINO ${ }^{1,3}$ \& JORGE L. WAECHTER ${ }^{4}$
}

\begin{abstract}
Summary: Rossado, A. J., P. L. Mai, J. M. Bonifacino \& J. L. Waechter. 2014. Acianthera hygrophila (Orchidaceae), new record for Uruguay. Bonplandia 23(2): 143-150.

The epiphytic orchid Acianthera hygrophila (Barb. Rodr.) Pridgeon \& M.W. Chase is reported for the first time for the flora of Uruguay. This species was found in a riverside forest of the Río Yaguarón in the East of Cerro Largo Department, near the border with Brazil. This record marks the Southern limit of distribution of the species. A description, an illustration and a distribution map of $A$. hygrophila are provided, as well as an identification key for Acianthera species found in Uruguay.
\end{abstract}

Key words: Acianthera, Cerro Largo, epiphyte, limit of distribution, orchid, Pleurothallidinae.

Resumen: Rossado, A. J., P. L. Mai, J. M. Bonifacino \& J. L. Waechter. 2014. Acianthera hygrophila (Orchidaceae), nuevo registro para Uruguay. Bonplandia 23(2): 143-150.

Se reporta por primera vez para la flora de Uruguay la orquídea epífita Acianthera hygrophila (Barb. Rodr.) Pridgeon \& M.W. Chase. La misma fue hallada en el bosque ribereño del Río Yaguarón en el este del departamento de Cerro Largo próximo al límite con Brasil. Este registro representa el límite sur de distribución de la especie. Se presenta una descripción ampliada, ilustración y mapa de distribución de A. hygrophila, así como una clave para la identificación de las especies de Acianthera presentes en Uruguay.

Palabras clave: Acianthera, Cerro Largo, epífita, límite de distribución, orquídea, Pleurothallidinae.

\section{Introducción}

El género Acianthera Scheidw. (Orchidaceae) se ubica dentro de la subfamilia Epidendroideae Kostel., tribu Epidendreae Lindl. y subtribu Pleurothallidinae Lindl. (Dressler, 1993; Pridgeon et al., 2005). Esta subtribu posee distribución Neotropical y abarca de un 15 a
$20 \%$ del total de las especies de orquídeas del mundo (Pridgeon et al., 2001). Está conformada por taxones mayoritariamente de hábito epífito, de crecimiento simpodial, con rizomas portando caulomas (caules no rizomáticos) sin pseudobulbo, teretes y unifoliolados, hojas conduplicadas, y ovario articulado con el pedicelo (Pridgeon et al., 2001; 2005). En

\footnotetext{
${ }^{1}$ Laboratorio de Sistemática de Plantas Vasculares, Facultad de Ciencias, Universidad de la República, Iguá 4225 , Montevideo, Uruguay. Correo electrónico: andres.rossado@gmail.com

${ }^{2}$ Licenciatura en Gestión Ambiental, CURE, Universidad de la República, Ruta 9 y Ruta 15, Rocha, Uruguay. Correo electrónico: patimaimo@gmail.com

${ }^{3}$ Laboratorio de Botánica, Departamento de Biología Vegetal, Facultad de Agronomía, Montevideo, Uruguay. Correo electrónico: bonifacinoj@fagro.edu.uy

${ }^{4}$ Departamento de Botânica, Universidade Federal do Rio Grande do Sul, Av. Bento Gonçalves, 9500, Prédio 43433, Porto Alegre, RS, Brasil. Correo electrónico: jorgew.bio@gmail.com
} 
particular Acianthera se caracteriza por la presencia de caulomas sin anillo, inflorescencias principalmente situadas en la zona apical de los caulomas y dos polinios cerosos (Gonçalves \& Waechter, 2007). Este género comprende alrededor de 230 especies (WCSP, 2014) distribuidas desde México (Espejo \& LópezFerrari, 2008) hasta el nordeste de Argentina (Schinini et al., 2008) y este de Uruguay, con un elevada riqueza específica en Brasil (Pridgeon \& Chase, 2001).

Tradicionalmente las especies pertenecientes a Acianthera estaban incluidas dentro del género Pleurothallis R. Br. s.l., (Luer, 1986), el cual resultó ser polifilético según los análisis moleculares de Pridgeon et al. (2001). En este estudio las especies de Pleurothallis subg. Acianthera (Scheidw.) Luer forman un grupo natural en la base de Pleurothallis s.l., por lo que Pridgeon \& Chase (2001) deciden rehabilitar el género Acianthera, creado en 1842 por Scheidweiler, con algunos cambios en su circunscripción.

Según Luer (2006) la especie Acianthera hygrophila (Barb. Rodr.) Pridgeon \& M. W. Chase, pertenece al género Arthrosia (Luer) Luer, antes considerado un subgénero de Pleurothallis (Luer, 1986). El género Arthrosia es actualmente aceptado por Schinini et al. (2008) y se diferencia del resto de Pleurothallis s.l. por tener un callus transversal en la base del labelo que articula con una cavidad transversal en la base de la columna (Luer, 1986). Sin embargo, en este trabajo optamos por seguir el criterio de Pridgeon $\&$ Chase $(2001 ; 2005)$ quienes incluyen a la especie dentro de Acianthera, ya que consideran que son necesarios más estudios filogenéticos antes de realizar nuevos cambios taxonómicos. A su vez, un estudio molecular reciente ubica a la especie dentro del género Acianthera sección Arthrosiae Chiron \& van den Berg. (Chiron \& van den Berg., 2012) junto a 12 especies más.

En Uruguay la familia Orchidaceae está representada aproximadamente por 60 especies, en su mayoría terrestres (Izaguirre, 2010). Únicamente nueve especies poseen hábito epífito (Izaguirre, 2013); ocho de éstas consideradas prioritarias para la conservación a nivel nacional, debido principalmente a su distribución restringida dentro del país (Marchesi et al., 2013). Entre los géneros epífitos se encuentra
Acianthera, único representante de la subtribu Pleurothallidinae en Uruguay, que de acuerdo con Izaguirre (2013) presenta dos especies: $A$. pubescens (Lindl.) Pridgeon \& M. W. Chase y $A$. sonderiana (Rchb. f.) Pridgeon \& M. W. Chase.

El presente trabajo tiene como objetivo reportar la presencia para Uruguay de una tercera especie de Acianthera, A. hygrophila, ampliando de este modo el conocimiento de la distribución de la especie y elevando a diez el número de orquídeas epífitas para el país. Se acompaña la nueva cita con una descripción detallada de la especie, datos de hábitat y fenología, ilustraciones y mapa de distribución, así como de una clave para la identificación de las especies del género Acianthera presentes en Uruguay.

\section{Material y Métodos}

Este trabajo se basa en el estudio de ejemplares de herbario depositados en ICN, CTES, MVFA, MVJB y MVM (acrónimos según Thiers, 2014). Se complementó el estudio de los ejemplares de herbario con la observación de plantas in situ y en cultivo. La identificación y confirmación de especímenes fue realizada a partir de bibliografía especializada de Uruguay y de la región (Gonçalves, 2005; Gonçalves \& Waechter, 2011; Izaguirre, 2013).

El mapa de distribución se elaboró a partir de los datos de ubicación contenidos en las etiquetas de los ejemplares examinados. Adicionalmente, para Brasil se incluyeron los datos de distribución reportados en trabajos anteriores para el género (Gonçalves, 2005; Rambo, 1965); y se incorporaron las exsiccatas con ubicación geográfica de Acianthera hygrophila disponibles en SpeciesLink (2014).

\section{Resultados}

Acianthera hygrophila (Barb. Rodr.) Pridgeon \& M. W. Chase, Lindleyana 16(4): 244. 2001. Fig. 1.

Pleurothallis hygrophila Barb.Rodr. Gen. Sp. Orchid. 1: 7. 1877. Tipo: Brasil. Minas Gerais: Ribeirão Machadinho, V, J. B. Rodrigues s.n. 
A. J. Rossado et al., Acianthera hygrophila, nuevo registro para Uruguay.

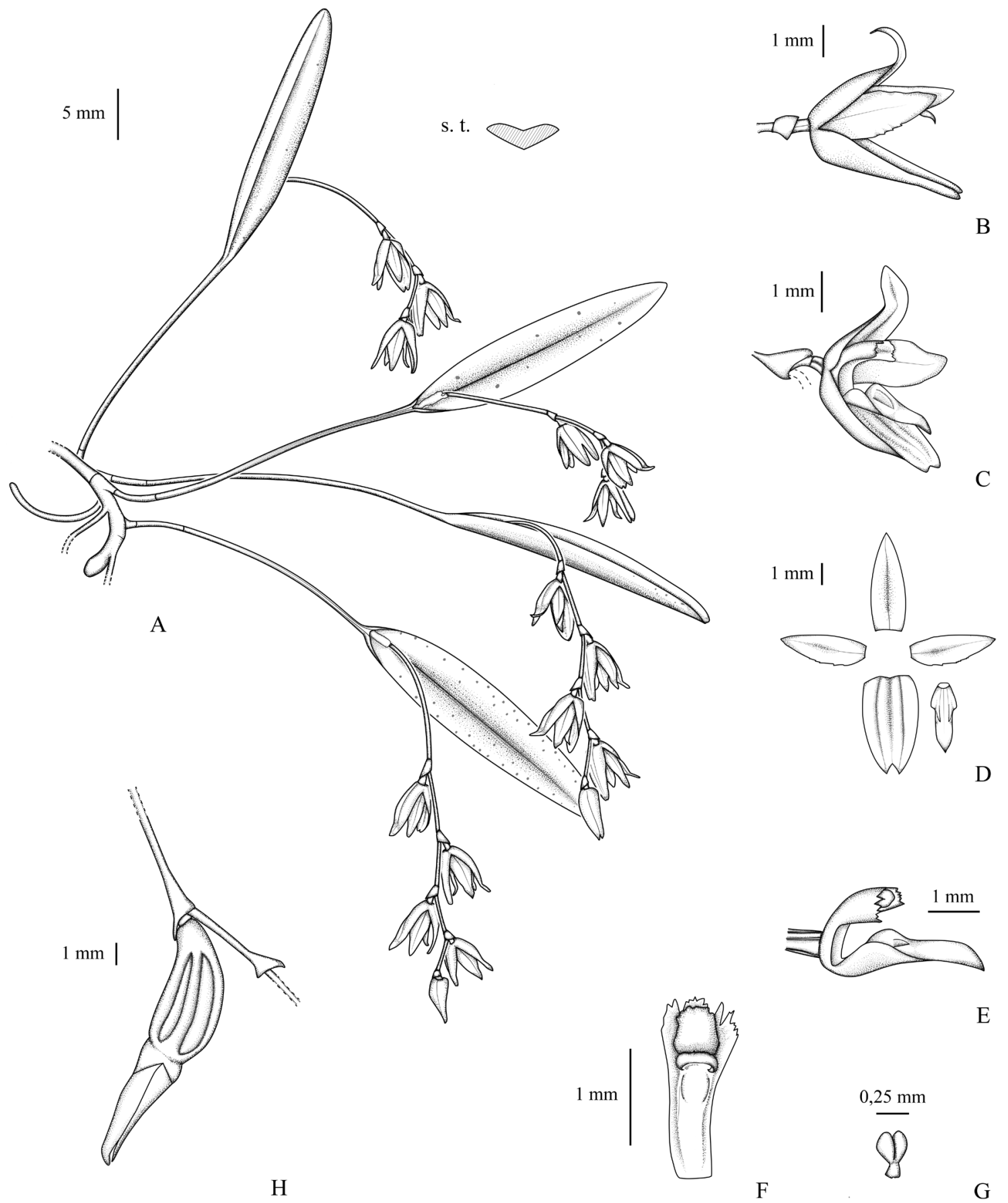

Fig. 1. Acianthera hygrophila. A: Hábito (s.t.: sección transversal de una hoja). B: Flor vista lateral. C: Flor vista lateralfrontal, con uno de los pétalos removido. D: Piezas florales extendidas. E: Ovario, columna y labelo en vista lateral. F: Columna vista ventral, se observa clinandrio, antera, rostelo y estigma. G: Polinario. H: Fruto con restos de perianto. (A-G: Rossado et al. 178, MVJB, MVFA; H: Rossado et al. 213, MVJB). Dibujó Andrés Rossado. 
(holotipo RB, destruido); neotipo: Brasil. Rio de Janeiro: Serra dos Órgãos, s.d., T. de Moura 14 (RB, designado por Gonçalves \& Waechter (2004), imagen RB 0542551!).

Plantas epífitas, pequeñas, formando matas densas. Raices filiformes, blanquecinas. Rizomas 1,1-1,6 mm de diámetro, cilíndricos, reptantes, adpresos al sustrato, cubiertos por vainas papiráceas-escariosas. Caulomas 9-54 mm long. $\times 0,5-0,6 \mathrm{~mm}$ lat., erectos, por lo general más largos que las hojas, distanciados 2,2-3,0 mm entre sí. Hojas 1,2$3,8 \mathrm{~cm}$ long. $\times 0,3-0,6 \mathrm{~cm}$ lat. $\times 0,15-0,3 \mathrm{~cm}$ de espesor, angostamente elípticas, ápice agudo, diminutamente tridentado, a veces aparentemente bidentado, base cuneada gradualmente engrosada con respecto al cauloma, planas a plegadas por el nervio medio, rectas a recurvas, carnosas, glabras, verde oscuras, frecuentemente con pigmentaciones violáceas circulares especialmente en la cara abaxial. Racimos 2-5 cm long., terminales, uno por cauloma, péndulos, con (2) 3-5 (7) flores distanciadas entre 3,0-6,5 mm entre sí. Brácteas florales ca. $1 \mathrm{~mm}$ long., membranáceas, cremas. Flores ca. $5 \mathrm{~mm}$ long. $\times 5 \mathrm{~mm}$ lat., membranáceas, glabras, crema adquiriendo tonalidades rojizas al madurar. Sépalo dorsal 4,5-5,0 mm long. $\times$ 0,9-1,1 $\mathrm{mm}$ lat., elíptico, ápice agudo y recurvo, base gibosa, membranáceo, línea media violácea. Sépalos laterales fusionados por más de $2 / 3$ de la longitud, 4,3-4,6 mm long $\times 1,1-1,4$ $\mathrm{mm}$ lat. cada uno, elípticos, rectos, ápice agudo, membranáceos, líneas medias leve a marcadamente violáceas. Pétalos 3,7-4,2 mm long. $\times 1,4-1,7 \mathrm{~mm}$ lat., elípticos a ovados, ápice agudo, margen entero a veces con pocos dientes diminutos, membranáceos (más que los sépalos), línea media violácea más notoria hacia la base. Labelo 2,9-3,5 mm long. $\times 1,4 \mathrm{~mm}$ lat., lingüiforme, trilobulado, ápice agudo y recurvo, articulado hacia la base con la columna, carnoso, verde claro a levemente amarillento; callosidad formada por dos crestas en la porción media del lóbulo central; lóbulos laterales erguidos, más pequeños que el central. Columna 1,7-2,1 mm long., arqueada, alada, verde clara; clinandrio denticulado irregularmente. Antera globosa, polinios 2, amarillos. Rostelo semicircular, estigma cóncavo, ventral. Frutos cápsula verde-rojiza, con restos persistentes de piezas florales en el ápice.

Distribución y hábitat: Especie citada para noreste de Argentina (Misiones; Schinini et al., 2008), Brasil (Amazonas, Maranhão, Minas Gerais, Espírito Santo, Rio de Janeiro, São Paulo, Paraná, Santa Catarina y Rio Grande do Sul; Gonçalves, 2005) y este de Uruguay (Cerro Largo) (Fig. 2). Generalmente presenta hábito epífito y ocasionalmente epipétrico (Gonçalvez, 2005). En el país hasta el momento ha sido registrada únicamente en el bosque ribereño del Río Yaguarón, donde se desarrolla como epífita formando matas densas sobre troncos y ramas principales de árboles de gran tamaño (especialmente de Luehea divaricata Mart.). En esta localidad fue observada en baja abundancia y desarrollándose a media sombra en lugares húmedos, junto a otras especies de orquídeas epífitas como Acianthera sonderiana, a la cual se asemeja vegetativamente (ver Obs. II). En Brasil A. hygrophila se encuentra ampliamente distribuida en la Mata Atlántica y además posee algunos registros en áreas de la Amazonia (Gonçalvez, 2005). Las colectas de Uruguay junto con la procedente de la localidad de Herval (Brasil) representan los registros más australes para la especie. Las otras dos especies de Acianthera componentes de la flora uruguaya poseen, al igual que $A$. hygrophila, distribución restringida al este del departamento de Cerro Largo, donde se ubica el límite sur de distribución del género y de toda la subtribu Pleurothallidinae. Por otra parte, la presencia de A. hygrophila reafirma la influencia de la flora atlántica y paranaense en el este del país, donde un conjunto considerable de taxones presentan su límite sur de distribución (Grela \& Brussa, 2003, 2005; Grela, 2004; Brussa \& Grela, 2007; Haretche et al., 2012).

Fenología y biología reproductiva: En Uruguay florece desde mediados y finales de julio a setiembre. Individuos con fruto fueron observados en setiembre. En un estudio realizado en Curitiba (Brasil; Santos Filho, 2007), se observó que cada flor permanece abierta de 9 a 13 días, son polinizadas por 


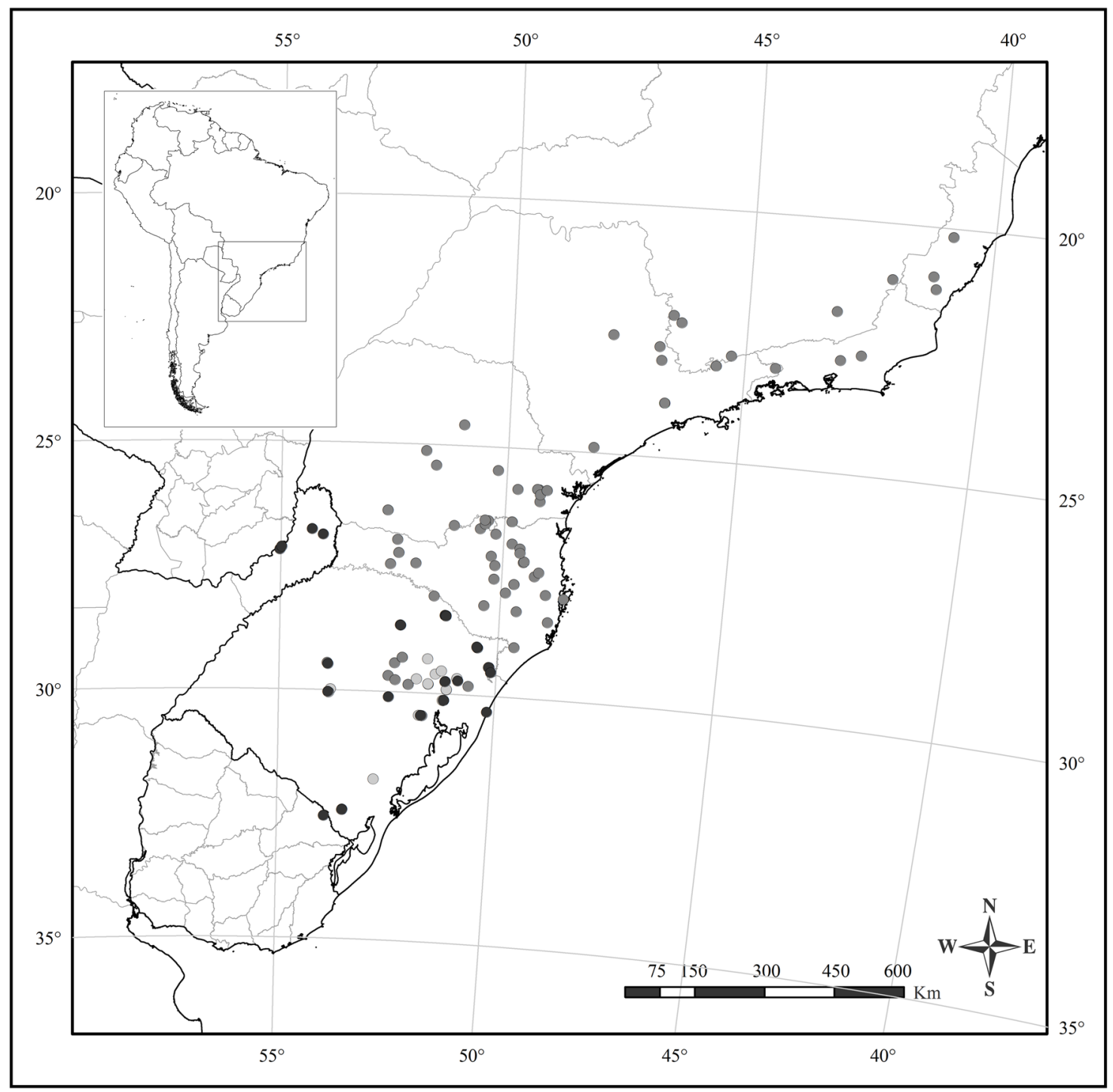

Fig. 2. Distribución geográfica de Acianthera hygrophila. Se muestra el área principal de distribución de la especie (se excluyen los registros de la zona norte de Brasil: estados de Amazonas y Maranhão). Referencias: círculos negros - ejemplares observados; círculos gris oscuros - ejemplares de SpeciesLINK (2014); círculos gris claros - ejemplares citados en Gonçalves (2005) y Rambo (1965).

dípteros de las familias Ceratopogonidae y Sciaridae, y que se trata de una especie autoincompatible.

Estado de conservación: Consideramos que Acianthera hygrophila debe ser incluida en el listado de especies prioritarias para la conservación en Uruguay, a causa de su reducida distribución dentro del mismo. A su vez esta especie habita bosques con árboles de gran desarrollo, por lo que su conservación depende en gran parte de la protección de este tipo de ambientes.

Obs. I: Los órganos vegetativos y reproductivos de las plantas de Acianthera hygrophila examinadas en Uruguay poseen dimensiones próximas a los valores inferiores, o incluso en algunos casos se encuentran por debajo de los rangos de medidas obtenidas 
por Gonçalves (2005) para la revisión de Acianthera de Rio Grande do Sul (Brasil) y de las medidas obtenidas en los materiales examinados en el ICN para el presente trabajo. Las diferencias se hacen especialmente notorias en los caracteres vegetativos como largo del cauloma y de la hoja.

Obs. II: En Uruguay Acianthera sonderiana es la única especie que se asemeja vegetativamente a $A$. hygrophila. La primera posee rizomas más cortos, hojas más carnosas y forma matas más compactas. Asimismo las hojas de $A$. sonderiana son verde claras en comparación con $A$. hygrophila de hojas verde oscuras, frecuentemente con pequeñas manchas violáceas distribuidas a lo largo de toda la lámina. Estas especies poseen diferencias morfológicas a nivel reproductivo que las hacen fácilmente identificables (ver clave). Adicionalmente, en Uruguay existen diferencias fenológicas entre ambas, $A$. hygrophila florece en invierno mientras que $A$. sonderiana florece desde finales de verano a mediados de otoño.
Material examinado: URUGUAY. Cerro Largo: Río Yaguarón, 22-II-2013 (en cultivo, prensado 29-VIII-2013), A. J. Rossado et al. 178 (MVFA, MVJB); Río Yaguarón, 22- IX-2013, A. J. Rossado et al. 213 (MVJB).

Material adicional examinado: ARGENTINA. Misiones: El Dorado, 18-V-1991, A. E. Johnson 193 (CTES); Libertador Gral. San Martín, 15-X-1990, $A$. E. Johnson 481 (CTES); San Pedro, 1-IV-1994, A. E. Johnson 538 (CTES). BRASIL. Rio Grande do Sul: Bom Jesus, s. d., J. Dutra 1109 (ICN); Cambará do Sul, 22-VI-2009, C. R. Buzatto 538 (ICN); Canela, 2-I-1973, J. Jung s.n. (ICN 21897); Eldorado do Sul, 24-V-2002, C. Giongo \& V. Knuppi (ICN 124925); Esmeralda, 14-V-1978, L. Arzivenco 50 (ICN); Esmeralda, 7-VI-1979, J. L. Waechter 1251 (ICN); Herval, 13-IV-2007, M. Grings 1008 (ICN); Mato Castelhano, 1-X-2005, C. R. Buzzato \& R. Busato 10506 (ICN); Nova Petrópolis, X-2003, M. Grings 607 (ICN); Santa Cruz do Sul, 9-V-1976, J. L. Waechter 252 (ICN); Santa Maria, 13-VI-1991, J. L. Waechter 2501 (ICN); São Leopoldo, 23-VI-1927, J. Dutra 986 (ICN); Tupanciretã, 3-II-2008, M. Grings 1286 (ICN).

\section{Clave para la identificación de las especies de Acianthera presentes en Uruguay}

1. Hojas mayores a $13 \mathrm{~mm}$ de ancho; flores pubescentes, carnosas.

A. pubescens

1 '. Hojas iguales o menores a $6 \mathrm{~mm}$ de ancho; flores glabras, membranáceas o cartáceas.

2. Hojas mayormente rollizas, base abruptamente engrosada con respecto al cauloma (en vivo). Inflorescencias erectas; flores amarillas, agrupadas en la porción apical de la inflorescencia; pétalos iguales o menores a la mitad del largo de los sépalos laterales.

A. sonderiana

$2^{\prime}$. Hojas mayormente planas a plegadas por el nervio medio, base gradualmente engrosada con respecto al cauloma (en vivo). Inflorescencias péndulas; flores crema, distribuidas a lo largo de la inflorescencia; pétalos mayores $\mathrm{a}^{3}{ }_{4}$ del largo de los sépalos laterales.

A. hygrophila

\section{Agradecimientos}

Deseamos agradecer a los curadores de los herbarios ICN, MVFA, MVJB y MVM por permitirnos estudiar los materiales depositados en las colecciones, a los curadores del herbario de CTES por poner a nuestra disposición imágenes escaneadas de ejemplares depositados en ese herbario. Agradecemos a Victoria Valtierra, María Zabaleta, CSIC, PEDECIBA,
Facultad de Ciencias y Facultad de Agronomía de la Universidad de la República por el apoyo brindado para la realización de las salidas de campo.

\section{Bibliografía}

BRUSSA, C. A. \& I. GRELA. 2007. Flora arbórea del Uruguay. Con énfasis en las especies de Rivera y 
Tacuarembó. Cofusa, Montevideo. 544 pp.

CHIRON, G. R. \& C. VAN DEN BERG. 2012. Révision taxonomique du genre Acianthera (Orchidaceae, Pleurothallidinae). Richardiana 12: 59-77.

DRESSLER, R. L. 1993. Phylogeny and classification of the orchid family. Cambridge University Press, Cambridge. 314 pp.

ESPEJO, A. \& A. R. LÓPEZ-FERRARI. 2008. Orquidáceas (Monocotiledóneas). En S. Ocegueda \& J. Llorente-Bousquets (Coords.), Catálogo taxonómico de especies de México, en Capital natural de México. V. 1: Conocimiento actual de la biodiversidad. Comisión Nacional para el Conocimiento y Uso de la Biodiversidad. México, D.F., México. CD1.

GONÇALVES, C. N. 2005. Estudos taxonômicos, morfológicos e biogegráficos em Acianthera (Orchidaceae). Tesis de Doctorado, Universidade Federal do Rio Grande do Sul, Porto Alegre, Brasil. $182 \mathrm{pp}$.

GONÇALVES, C. N \& J. L. WAECHTER. 2004. Notas taxonómicas e nomenclaturais em espécies brasileiras de Acianthera (Orchidaceae). Hoehnea 31: 113-117.

_ \& _ 2007. Sinopse histórica do gênero Acianthera (Pleurothallidinae, Orchidaceae) e razões para o seu reestabelecimento. Orquidário 21: 22-29.

— \& - 2011. Sinopse do gênero Acianthera Scheidw. (Orchidaceae) no Rio Grande do Sul, Brasil. Revista Brasileira de Biociências 9: 143155.

GRELA, I. 2004. Geografía florística de las especies arbóreas de Uruguay: propuesta para la delimitación de dendrofloras. Tesis de Maestría, PEDECIBAUniversidad de la República, Montevideo, Uruguay. $97 \mathrm{pp}$.

\& C. A. BRUSSA. 2003. Relevamiento florístico y análisis comparativo de comunidades arbóreas de Sierra de Ríos (Cerro Largo - Uruguay). Agrociencia Uruguay 7: 11-26.

— — . 2005. Sinningia macrostachya (Lindl.) Chautems, nuevo registro de Gesneriaceae para la flora del Uruguay. Iheringia Sér. Bot. 60: 249-252.

HARETCHE, F., P. MAI \& A. BRAZEIRO. 2012. Woody flora of Uruguay: inventory and implication within the Pampean region. Acta Bot. Brasil. 26: 537-552.

IZAGUIRRE, P. 2010. Novedades en orquídeas para Uruguay: primera contribución. Agrociencia Uruguay 14: 1-9.

—. 2013. Novedades en orquídeas para Uruguay: segunda contribución. Plantas epífitas. Agrociencia Uruguay 17: 22-35.

LUER, C. A. 1986. Icones Pleurothallidinarum III. Systematics of Pleurothallis (Orchidaceae). Monogr. Syst. Bot. Missouri Bot. Gard. 20: 1-107.
LUER, C. A. 2006. Icones Pleurothallidinarum XXVIII. Miscellaneous new taxa in the Pleurothallidinae (Orchidaceae). Monogr. Syst. Bot. Missouri Bot. Gard. 105: 245-259.

MARCHESI, E., E. ALONSO, L. DELFINO, M. GARCÍA, F. HARETCHE \& C. A. BRUSSA. 2013. Plantas Vasculares. En A. Soutullo, C. Clavijo, \& J. A. Martínez-Lanfranco (eds). Especies prioritarias para la conservación en Uruguay. Vertebrados, moluscos continentales y plantas vasculares, pp. 27-71. SNAP/DINAMA/MVOTMA y DICYT/ MEC, Montevideo.

PRIDGEON, A. M. \& M. W. CHASE. 2001. A phylogenetic reclassification of Pleurothallidinae (Orchidaceae). Lindleyana 16: 235-271.

__, R. SOLANO \& M. W. CHASE. 2001. Phylogenetic relationships in Pleurothallidinae (Orchidaceae): combined evidence from nuclear and plastid DNA sequences. Amer. J. Bot. 88: 2286-2308.

, P. J. CRIBB, M. W. CHASE \& F. N. RASMUSSEN (Eds). 2005. Genera Orchidacearum. V. 4: Epidendroideae (Part 1). Oxford University Press, Oxford. 696 pp.

RAMBO, B. 1965. Orchidaceae Riograndenses. Iheringia Sér. Bot. 13: 1-96. (Obra póstuma).

SANTOS FILHO, J. F. 2007. Polinização e biología reprodutiva de três espécies do gênero Acianthera Scheidw. (Orchidaceae) em floresta ombrófila mista. Tesis de Maestría, Universidade Federal do Paraná, Curitiba, Brasil. 32 pp.

SCHININI, A., J. L. WAECHTER, P. IZAGUIRRE \& C. LEHNEBACH. 2008. Orchidaceae. En F. O. Zuloaga, O. Morrone \& M. J. Belgrano (eds.). Catálogo de las plantas vasculares del Cono Sur (Argentina, Sur de Brasil, Chile, Paraguay y Uruguay), pp 472-609. St. Louis, Missouri Botanical Garden.

SPECIESLINK. 2014. Herbário da Universidade Federal de Minas Gerais (BHCB), Herbário do Departamento de Botânica da Universidade Federal de Santa Catarina (FLOR), Herbário da Universidade Estadual de Londrina (FUEL), Herbário Dr. Roberto Miguel Klein (FURB), Herbário da Universidade Tecnológica Federal do Paraná Campus Campo Mourão (HCF), Herbário do Vale do Taquari (HVAT), Herbário do Instituto de Ciências Naturais (ICN), Herbário do Parque da Ciência Newton Freire Maia (IRAI), Missouri Botanical Garden - Brazilian records (MOBOT BR), Herbário Dimitri Sucre Benjamin (RB), Herbário do Estado "MariaEneyda P. Kaufmann Fidalgo” - Coleção de Fanerógamas (SP), Herbário do Departamento de Botânica (UPCB). Disponible: $<$ http://www.splink.org.br $>$ [Consulta: III-2014].

THIERS, B. 2014 [permanentemente actualizado, 
consulta 14-II-2014]. Index Herbariorum: A global directory of public herbaria and associated staff. New York Botanical Garden's Virtual Herbarium. Disponible: $<$ http://sweetgum.nybg.org/ih.
WCSP. 2014. World Checklist of Selected Plant Families. Facilitated by the Royal Botanic Gardens, Kew. Disponible: http://apps.kew.org/wcsp/ [Consulta:14-II-2014].

Original recibido el 29 de julio de 2014; aceptado el 30 de octubre de 2014. 